To be published in The Astrophysical Journal, Feb. 101996

\title{
MAGNETIC AND SPIN HISTORY OF VERY YOUNG PULSARS
}

\author{
Alexander Muslimov日 \\ Institut für Astrophysik der Universität Bonn, Auf dem Hügel 71, 53121 Bonn, Germany \\ muslimov@astro.uni-bonn.de \\ and \\ Dany Page \\ Instituto de Astronomía, UNAM, Apdo Postal 70-264, 04510 México D.F., México. \\ page@astroscu.unam.mx
}

\begin{abstract}
After Michel (1994) introduced a phenomenological picture of "rapid magnetization" of newly born neutron stars (NSs), Muslimov \& Page (1995) suggested that the physical conditions accompanying the formation of a NS (convection in the NS envelope, high-rate accretion in a supernova) may result in the large scale surface magnetic field of the NS having a low value $\left(\sim 10^{8}-10^{9} \mathrm{G}\right)$, while the bulk of the magnetic flux is submerged under the stellar surface. The subsequent growth of the large scale surface magnetic field then occurs owing to ohmic diffusion of a strong internal field $\left(\sim 10^{12}-10^{13} \mathrm{G}\right)$ and proceeds over a timescale of $10^{2}-10^{3} \mathrm{yr}$, depending on the early thermal history of the NS, initial distribution of magnetic flux, and electrical resistivity in the crust. Referring to this suggestion, we perform numerical computations to demonstrate its possible relevance to young radio pulsars. In our calculations, we use different NS structures based on a model of dense matter presenting a phase transition to kaon condensation which softens the equation of state. This model implies both slow and fast neutrino cooling, depending on the stellar mass (which is assumed to be in the range of $1.35-1.50 M_{\odot}$ ). We present sequences of secular evolution of the surface magnetic field, spin-down luminosity, spin period, "braking index," and spin-down age best matching the
\end{abstract}

\footnotetext{
${ }^{1}$ Alexander von Humboldt Fellow
} 
corresponding measured and derived quantities for PSR 0531+21 (Crab), PSR 0540-69, and PSR 1509-58. We conclude that the effect under consideration reproduces remarkably well both the observed rotational characteristics and derived values of the surface magnetic field strength for these radio pulsars, the only ones having reliable measurements of the braking indices. In addition, our analysis suggests that NSs in the Crab and PSR 0540-69 have experienced fast neutrino cooling and that their masses are above the critical mass for the phase transition while the NS in PSR 1509-58 has a mass below this critical mass and has therefore undergone slow neutrino cooling.

Subject headings: $\quad$ pulsars: individual (PSR 0531+21, PSR 0540-69, PSR 1509-58) — stars: neutron — stars: magnetic fields 


\section{INTRODUCTION}

The timing of radio pulsars reveals that their "clock mechanisms" are subject to a systematic delay (spinning down) and that various irregularities in their run such as "glitches," "microglitches," etc., may occur. The measurements of the first and second time derivatives of pulsars' spin period, $\dot{P}$ and $\ddot{P}$, respectively, can provide us with invaluable information about the rotational dynamics of neutron stars (NSs). For the standard formula describing the secular decrease in the angular velocity $\Omega$ of an NS,

$$
\dot{\Omega} \propto-\Omega^{n}
$$

where $n=3$ corresponds to the mechanism of pure magnetic dipole braking, the "braking index" (see Manchester \& Taylor 1977, hereafter MT, eq. [6-17]),

$$
n \equiv \frac{\Omega \ddot{\Omega}}{(\dot{\Omega})^{2}},
$$

can, in principle, be determined from observations. In practice, the measurements of braking indices are contaminated by the effects of "restless" behavior of $\dot{P}$ over short timescales and are a point of controversy. Only three young pulsars have measured values of $n$ not dominated by timing noise: PSR 0531+21 (Crab), $n=2.509 \pm 0.001$ (Lyne, Pritchard, \& Smith 1988); PSR 0540-69, $n=2.01 \pm 0.02$ (Nagase et al. 1990; Gouiffes, Finley, \& Ögelman 1992); and PSR 1509-58, $n=2.837 \pm 0.001$ (Kaspi et al. 1994). The observed rotational properties of these three pulsars are listed in Table 1 .

Theoretically, as is summarized in MT (see Table 9-1), there are a number of factors affecting the braking index, e.g., multipole electromagnetic radiation $(n \geq 5)$, gravitational quadrupole radiation $(n=5)$, magnetic field decay $(n>3)$, radial deformation of field lines $(1 \leq n \leq 3)$, pulsar wind $(n<3)$, relaxation of the NS equilibrium form $(n<3)$, pulsar transverse velocity $(n<3)$, etc. To these, one should add two mechanisms recently proposed: spin-up of the NS during its early evolution owing to the intense neutrino emission $(n<0)$ (Alpar \& Ögelman 1990) and crustal "plate" motions (Ruderman 1991), the latter being able to produce values of $n$ larger or smaller than 3 . Also, Blandford \& Romani (1988) have discussed the possibility that the braking indices of PSR $0531+21$ and PSR 1509-58 may be consistent with a secular growth of their magnetic field. They appealed to the thermomagnetic instability in the NS crust (Blandford, Applegate, \& Hernquist 1983, see also Urpin, Levshakov, \& Yakovlev 1986) as a basic reason for the magnetic field growth. Finally, Michel (1991) has also emphasized that the monotonic increase of the magnetic field with time would give braking indices less than 3 in all young pulsars where it can be determined. 
In this paper we employ our recent calculations of growth of the surface magnetic field in a newly born NS (Muslimov \& Page 1995, hereafter MP) to demonstrate the striking agreement of our model with the available observational data for the Crab, PSR 0540-69, and PSR 1509-58. Our tentative analysis (see MP) had been stimulated by a phenomenological picture recently discussed by Michel (1994, hereafter M94) that the magnetic field of a NS at birth is low and increases, by a factor of $\gtrsim 10^{3}$, to the typical value of $\sim 10^{12} \mathrm{G}$ during a period of a few hundred years following the supernova. MP suggested, as a physical basis for this picture, that the surface magnetic field of a young NS may grow from a very low value $\left(\sim 10^{8}-10^{9} \mathrm{G}\right)$ owing to ohmic diffusion of a strong internal magnetic field ( $\gtrsim 10^{12} \mathrm{G}$ ) that was initially submerged under the surface layers. Assuming that an NS spins down as a result of magnetic dipole radiation, we determine the free fitting parameters of our model. For example, for each of these pulsars we specify the initial spin period and initial profile of the magnetic flux in the crust.

These early magnetic and spin evolutions are strongly affected by the thermal evolution of the NS. In the present study we exploit different stellar structures and thermal histories to fit the data for the three young pulsars. Specifically, we consider different NS masses and the kaon condensation phase transition (Thorsson, Prakash \& Lattimer 1994; hereafter TPL) in the NS core which results in different cooling histories depending on the mass. Within the framework of our analysis, the fast cooling of an NS provides the best fit for the Crab and PSR 0540-69, including accounting for the discrepancy between their "real" and

spin-down ages. In the case of PSR 1509-58, we will need a slow cooling, i.e., a star of mass below the critical mass for the phase transition.

In $\S 2$ we present our numerical model, and in $\S 3$ we illustrate matching of our calculations with the observed parameters for PSR 0531+21 (Crab), PSR 0540-69, and PSR 1509-58. Our principal conclusions are summarized in $\S$.

\section{BASIC ASSUMPTIONS AND METHOD}

\subsection{General Picture}

We assume that an NS is born with a spin period in the range 20-40 ms. Alternatively (see, e.g., M94), the luminosities of the NSs born as rapid rotators will become large before the NSs slow down to the present luminosities of the Crab, PSR 0540-69, and PSR 1509-58 which are already large enough. To calculate the spin evolution of the NS we use the standard formula (see, e.g., MT, p. 110, 180) for the spin-down torque resulting from emission of magnetic dipole radiation. 
If the secular evolution of the dipole magnetic moment of an NS is the only physical reason affecting its spinning down, it is straightforward to derive the following expression for the braking index

$$
n=3+2\left(\frac{\dot{B}_{\text {surf }}}{B_{\text {surf }}}\right)\left(\frac{\Omega}{\dot{\Omega}}\right),
$$

where $B_{\text {surf }}$ is the surface value of the magnetic field strength at the magnetic pole. (A similar formula, but missing a factor 2 in the second term, has been used by Chanmugam \& Sang [1989] in their analysis of the possible response of braking indices to ohmic field decay in pulsars.)

In formula (3) $\dot{\Omega}$ is always negative and, depending on whether $\dot{B}_{\text {surf }}>0$ (field growth) or $\dot{B}_{\text {surf }}<0$ (field decay), we have $n<3$ or $n>3$, respectively. Here we attempt to quantitatively examine the possibility that, in a newly born NS, the magnetic field (of strength $\sim 10^{12}-10^{13} \mathrm{G}$ ), initially trapped under the stellar surface, diffuses ohmically up through the crust ( MP). The main uncertainty in this picture is the form of the initial distribution of the magnetic flux in the upper crust. For illustrative purposes, we describe the initial profile of magnetic flux in the crust by referring to the depth down to which the flux is submerged under the stellar surface. Approximately at this depth the magnetic flux has a maximum value and decreases by a factor of $\sim 10^{2}-10^{3}$ toward the stellar surface.

\subsection{Basic Equations and Solution}

We now consider the evolution of the dipole component of a purely poloidal magnetic field $\mathbf{B}$ in the NS crust. For a dipole mode, in spherical coordinates $(r, \theta, \phi)$, we can express $\mathbf{B}$ in the form

$$
\mathbf{B}=B_{0}\left(\frac{S}{r^{2}} \cos \theta \mathbf{e}_{r}-\frac{1}{2 r} \frac{\partial S}{\partial r} \sin \theta \mathbf{e}_{\theta}\right),
$$

where $B_{0}$ is some normalization magnitude of the field, $\mathbf{e}_{r}$ and $\mathbf{e}_{\theta}$ are unit vectors in the radial and meridional directions, and $S=S(r, t)$ is the stream function.

Using the standard equation for the magnetic field evolution in flat spacetime (neglecting the hydrodynamic motions, thermomagnetic effects, and anisotropy of the electrical conductivity),

$$
\frac{\partial \mathbf{B}}{\partial t}=-\frac{c^{2}}{4 \pi} \nabla \times\left(\frac{1}{\sigma} \nabla \times \mathbf{B}\right),
$$

we arrive at the following equation for the evolution of $S$ :

$$
\frac{\partial S}{\partial t}=\frac{c^{2}}{4 \pi \sigma}\left(\frac{\partial^{2} S}{\partial r^{2}}-\frac{2 S}{r^{2}}\right),
$$


where $\sigma=\sigma(r, t)$ is the electrical conductivity of matter.

We solve equation (6) numerically subject to the appropriate boundary conditions at the stellar surface and at the base of the crust. At the surface $(r=R)$ we impose the standard boundary condition that the internal field merges continuously with an external vacuum field. For a dipole field this condition reads

$$
R \frac{\partial S}{\partial r}+S=0
$$

At the base of the crust $\left(r=r_{b}\right)$ we assume that

$$
S=\text { Const }
$$

This second condition is perfectly justified for our problem, and it means that the bulk of the magnetic flux [the magnetic flux through a hemisphere of radius $r$ is proportional to the value of the function $S(r, t)$ ] is frozen into the inner crust (outer core), at least over a timescale of $\gtrsim 10^{6} \mathrm{yr}$. The ohmic diffusion and decay of the magnetic field in the innermost layers of the crust occur on a much longer timescale and are of no importance for our present purpose. Note that in our calculations the conductivity of the crust depends on both the density and temperature of matter (see, e.g., MP and references therein for a relevant discussion of the conductivity regimes in the crust and corresponding numerical estimates), the latter changing with time as the NS cools down.

We perform our calculations of the spin-down luminosity, spin period, braking index, and spin-down age $\left(\tau_{s d}=P / 2 \dot{P}\right)$ of the NS by solving self-consistently the system of equations describing both its magnetic and spin evolution (owing to magnetic dipole braking). We also employ formula (3) in our field evolution code to calculate the secular evolution of the braking indices.

The principal parameters needed to be specified in our computations are the initial spin period of the NS, $P_{0}$, and the initial surface value of the magnetic field strength, $B_{\text {surf }}^{0}$. We assume that for the Crab, PSR 0540-69, and PSR 1509-58 the value of $B_{\text {surf }}^{0}$ is in the range of $(1-5) \times 10^{10} \mathrm{G}$, while $P_{0}$ ranges from $\sim 20$ to $\sim 40 \mathrm{~ms}$. It is important that our choice of $P_{0}$ for the Crab and PSR 0540-69 is not arbitrary, and we shall discuss this below.

Our calculations show clearly that the character of early magnetic and spin evolutions of a NS is determined by the dynamics of freezing and the structure of its crust. For example, the stellar models allowing for a rapid cooling result in a power-law temporal growth of the surface value of the magnetic field strength in the saturation regime. In contrast, the field evolution in the saturation regime for a relatively slowly cooling NS is more complex and cannot be described by a simple power-law time dependence. Also, 
the fact that the NSs in the Crab and probably in PSR 0540-69 have spin-down ages well exceeding their real ages can be understood naturally within the framework of our analysis only if we assume that these NSs have undergone a stage of rapid neutrino cooling. The corresponding stellar models and thermal histories we have employed in our calculations for these pulsars do reproduce remarkably well the discrepancy between their spin-down ages $\tau_{s d}$ and expected real ages $t_{\text {model }}$ (see $\S$ 3). Finally, in the case of PSR 1509-58 our results may testify that the spin-down age of the associated NS is less than its real age and that this can be attributed to relatively slow neutrino cooling of the NS.

The standard equation describing the evolution of the spin period owing to magnetic dipole losses can be easily integrated to give

$$
P=P_{0}\left(1+\alpha \int_{0}^{t} B_{\text {surf }}^{2} d t^{\prime}\right)^{1 / 2}
$$

where $\alpha$ is a numerical factor depending on the radius, moment of inertia, and initial value of the spin period of the NS. Using the above expression, we can represent the spin-down age of an NS in the form

$$
\tau_{\text {sd }} \equiv \frac{P}{2 \dot{P}}=\frac{K}{B_{\text {surf }}^{2}} \int_{0}^{t} B_{\text {surf }}^{2} d t^{\prime},
$$

where $K=\left[1-\left(P_{0} / P\right)^{2}\right]^{-1}$. Note that in our problem the integral $\int_{0}^{t} B_{\text {surf }}^{2} d t^{\prime}$ is dominated by the contribution from the saturation regime, and the integration can therefore be performed over the interval of time during which the field has been saturating. As we have emphasized above, for the stellar models with a rapid neutrino cooling (that have used for NSs in the Crab and PSR 0540-69) the surface value of the magnetic field strength increases in the saturation regime according to a power low. Thus, assuming that in the saturation regime, for NSs in the Crab and PSR 0540-69, $B_{\text {surf }} \propto t^{\epsilon}$, we obtain

$$
\tau_{s d} \sim \frac{K}{2 \epsilon+1} t
$$

and

$$
n \sim 3-\frac{2 \epsilon}{2 \epsilon+1} K .
$$

From equations (11) and (12) we find that

$$
K \sim 3-n+\frac{\tau_{s d}}{t}
$$

and

$$
\epsilon \sim \frac{3-n}{2(n-3+K)}
$$


We can use the expressions (13) and (14) for rough estimates of the values of $P_{0}$ and $\epsilon$ for the Crab and PSR 0540-69. Substituting the values $P, n, \tau_{s d}$, and $t=t_{\text {model }}$ into equations (13) and (14), we find that for these pulsars $P_{0} \sim 23 \mathrm{~ms}$ and $\epsilon \sim 0.18$, and $P_{0} \sim 41 \mathrm{~ms}$ and $\epsilon \sim 0.27$, respectively. These estimates justify our choice of the range of the initial spin periods for the Crab and PSR 0540-69. However, the above reasoning is not applicable to the NS in PSR 1509-58 because of the needed relatively slow stellar cooling and, as a consequence of this, more complex temporal evolution of the surface value of its field strength in the saturation regime. In this sense, our choice of $P_{0}$ for PSR 1509-58 is quite arbitrary.

For a given range of variation of $P_{0}$ and $B_{\text {surf }}^{0}$, we survey different profiles of the initial distribution of the magnetic flux in the crust to fit the observed and derived parameters such as $P, n, \tau_{s d}$, and $B_{\text {surf }}$. As a result of such a fitting we are able to determine the sensible range of the initial depths of submergence $\left(z_{s u b}\right)$ of the magnetic field in the crust (see Table 1). Note that the rate of spinning down and the value of the braking index decrease with increasing the initial depth of submergence $z_{\text {sub }}$ of the flux (for a given NS structure and thermal evolution), with the values of braking indices being most sensitive to the variation of this depth as a parameter. In our calculations, the initial profile and depth of submergence of the magnetic flux in the crust are therefore determined by matching the theoretical and measured values of braking indices for a given (derived) spin-down age of the pulsar. Note also that the variation of $P_{0}$ only slightly affects the calculated values of the spin period and braking index for relatively long-period pulsars such as PSR 1509-58.

\subsection{Neutron Star Models and Cooling}

Our many trials to fit the observed parameters of the three young pulsars led us to focus on different NS thermal histories and stellar structures. To perform a consistent analysis, we are thus forced to assume that the three NSs have different masses and that the equation of state (EOS) allows fast or slow neutrino cooling depending on the mass.

A number of emission mechanisms have been proposed for the fast neutrino cooling (see, e.g., Pethick 1992, Page 1994). In this paper, we adopt the possibility of kaon condensation which is presently the scenario most seriously discussed in the literature (see Brown \& Rho 1995 for a review). To build a consistent EOS, we follow the method of TPL, in which the effect of kaon condensation is superposed on an underlying purely baryonic EOS in the mean field approximation (very similar results have been obtained with a different formalism by the Kyoto group [see, e.g., Tatsumi 1995]). Due to the large uncertainties in the structure of matter at the densities characteristic of NS cores, we have 
many free choices. We select the baryonic EOS in a standard way, while we parameterize the kaon condensation properties to satisfy our needs, namely:

1. We select, as TPL did, the baryonic EOS from the set of parametric models of Prakash, Ainsworth \& Lattimer (1988, hereafter PAL): we use the model "PAL33" with an incompressibility $K_{0}=240 \mathrm{MeV}$ and a symmetry energy function $F(u)=u^{1 / 2}$ (where $u=n / n_{0}, n$ is the baryon number density and $n_{0}=0.16 \mathrm{fm}^{-3}$ is the "saturation" density). This EOS is intermediate in stiffness between the standard microscopic non relativistic FP EOS (Friedman \& Pandharipande 1981) and the microscopic relativistic MPA EOS (Müther, Prakash \& Ainsworth 1987). It provides a proton fraction that is low enough to prevent the fast neutrino emission by the direct Urca process (Lattimer et al. 1991). The mass-radius relationships for these three EOS are shown in Figure 1.

2. The properties of the kaon condensate in the formalism developed by TPL depend strongly on the values of the coupling constants of the model Lagrangian, all of them but one being fortunately constrained by experiments. The only poorly constrained parameter is thus $a_{3}$ (expressed as $a_{3} m_{s}$, where $m_{s}$ is the mass of the strange quark), and its reasonable range of variation is from $-134 \mathrm{MeV}$ down to about $-300 \mathrm{MeV}$ (see TPL). Given the underlying baryonic EOS, the value of $a_{3} m_{s}$ determines the critical density for the condensation phase transition, i.e., the critical mass for an NS to contain a kaon condensate core. Note that the maximum NS mass also depends on $a_{3} m_{s}$ but not so sensitively as the critical mass. We adopt in our calculations the value of $a_{3} m_{s}=-190 \mathrm{MeV}$. The resulting mass-radius relationship and the density profiles for five models of different masses are plotted in Figures 11 and 2. The NSs with masses below $1.37 M_{\odot}$, the critical mass for appearance of the condensate, are "standard" NSs, while more massive ones should rather be called "nuclear" stars (Brown \& Bethe 1994), since they contain roughly equal amounts of protons and neutrons, as laboratory nuclei do, and a very strong kaon condensate (unlike laboratory nuclei).

The motivation for our particular choice of $a_{3} m_{s}=-190 \mathrm{MeV}$ is twofold: first, it provides a transition from the slow to the fast neutrino cooling at a mass of $1.37 M_{\odot}$, which is within the range of measured NS masses (van Kerkwijk, van Paradijs \& Zuiderwijk 1995). Second, it gives a maximum mass of $1.575 M_{\odot}$ that is still consistent with the data and is also compatible with the possibility that the NS formed in SN 1987A went into a black hole (Bethe \& Brown 1995).

With regard to the cooling we come up with the following scenario. A NS of mass $M<1.37 M_{\odot}$ allows only the slow modified Urca process along with the associated nucleon bremsstrahlung processes and therefore follows the "standard" cooling scenario . If $M>$ $1.37 M_{\odot}$, then the presence of the kaon condensate increases the neutrino emission by 
orders of magnitude (Brown et al. 1988) resulting in fast cooling (Page \& Baron 1990). Above $1.463 M_{\odot}$ the direct Urca process is also allowed and dominates the total neutrino emissivity that results in an even faster cooling (Page \& Applegate 1992). The cooling curves for five models are shown in Figure 3, where the occurrence of the three different scenarios is clearly seen. The evolution of the profiles of internal temperature for two of these models is shown in Figure 4 . The profiles have strong gradients during the early phases of cooling, but after a few years the crust temperature in the density range $10^{11}$ - $10^{14} \mathrm{~g} \cdot \mathrm{cm}^{-3}$, where the currents producing the magnetic field are located, is uniform to within $50 \%$ or better.

Some details of the method used to produce these cooling models can be found in Page \& Baron (1990) and Page \& Applegate (1992). In this paper we introduce some modifications, which, besides the changes in the EOS, are as follows. We use a proper treatment of the suppression of the specific heat and neutrino emission by baryon pairing according to Levenfish \& Yakovlev (1994a,b). For the kaon condensate neutrino emissivity we use the formula

$$
\epsilon_{\nu}^{K}=7.6 \times 10^{25} \times T_{9}^{6}\left(\frac{m_{n}^{*}}{m_{n}}\right)^{2}\left(\frac{\mu_{e}}{m_{\pi}}\right) \frac{\sin ^{2} \theta_{K}}{2}\left[1+3\left(g_{A}^{\prime}\right)^{2}\right] \sin ^{2} \theta_{C} \mathrm{erg} \mathrm{cm}^{-3} \mathrm{~s}^{-1},
$$

which is obtained from the similar formula of Brown et al. (1988) by replacing the $\theta_{K}^{2}$ by $\sin ^{2} \theta_{K}$ to take into account that $\theta_{K}$ is not small. Also, an additional factor of 2 has been included following Thorsson et al. (1995). In the above formula $m_{n}$ and $m_{n}^{*}$ are, respectively, the neutron free and effective masses; $\mu_{e}$ is the electron chemical potential; $m_{\pi}$ is the pion mass; $g_{A}^{\prime} \sim 1$ is the in-medium Gamow-Teller coupling constant; $\theta_{C}=13^{\circ}$ is the Cabbibo angle; $T_{9}$ is the temperature in $10^{9} \mathrm{~K}$; and $\theta_{K}$ is the chiral angle characterizing the kaon condensate strength $\left(\theta_{K}\right.$ grows rapidly to near $90^{\circ}$ above the critical density). We have incorporated nucleon pairing by employing the results of, correspondingly, Ainsworth, Wambach, \& Pines (1989) [for neutron ${ }^{1} \mathrm{~S}_{0}$ pairing (inner crust)], Takatsuka (1972) [for neutron ${ }^{3} \mathrm{P}_{2}$ pairing (core)], and Takatuska (1973) [for proton ${ }^{1} \mathrm{~S}_{0}$ pairing (core)]. These core neutron and proton pairings (see Page 1994 for a comparative presentation of the relevant calculations) do not affect our computations because at the densities typical for the inner core (where the intense neutrino emission occurs) the corresponding critical temperatures $T_{c}$ are vanishingly low and do not allow the pairings. In contrast, neutron pairing in the inner crust is more important, since it reduces substantially the specific heat in this region, where the main currents producing the stellar magnetic field are located. As a result, the cooling of the crust proceeds faster during the first few decades. Note that the calculation of Ainsworth et al. (1989) is the most accurate treatment available to date and gives $T_{c}$ of the order of $10^{10} \mathrm{~K}$ in most of the inner crust. The uncertainty in the profile of $T_{c}$ does not affect our results (as can be seen from Fig. 4), since the temperature of the 
inner crust drops below $10^{10} \mathrm{~K}$ very rapidly (within a timescale much shorter than that for the magnetic field evolution in the inner crust).

\section{RESULTS OF NUMERICAL CALCULATIONS AND DISCUSSION}

The three observables are $P, \dot{P}$, and $\ddot{P}$, or equivalently $P, \tau_{s d}$ (and $B_{\text {surf }}$ with redundancy), and $n$. These observables are listed in Table 1 together with the parameters of our models. Our models aim at fitting these three (four with $B_{\text {surf }}$ ) observed values at a given model age $t_{\text {model }}$ as listed in Table 1. The age of the Crab pulsar is known, and the $t_{\text {model }}$ is taken to be equal to this age. In the case of PSR 0540-69, the study of the associated supernova remnant (SNR 0540-69) indicates an age $\tau_{S N R} \sim 760 \mathrm{yr}$ (Kirshner et al. 1989), but the actual age may be somewhat larger or smaller depending on whether the remnant's expansion has been accelerated by the pulsar or slowed down by the interaction with the interstellar medium. We thus only impose that $t_{\text {model }} \sim \tau_{S N R}$. The age of PSR 1509-58 is highly uncertain, and $t_{\text {model }} \sim 1,860 \mathrm{yr}$ is an output of our fit for this pulsar. If one accepts the association of PSR 1509-58 with the supernova SN 185 (Thorsett 1992), then this age is very close to the real age (however, Strom [1994] criticized this association).

We show in Figure 5 the temporal evolution of the surface magnetic field $B_{\text {surf }}$ (strictly speaking, its component perpendicular to the spin axis), spin-down luminosity $L_{s d}$, spin period $P$, braking index $n$, and spin-down age $\tau_{s d}$ for three different models. The present positions (i.e., at age $t_{\text {model }}$ ) on the evolutionary tracks are marked by dots. Figures $5 \mathrm{~A}$, $5 \mathrm{~B}$, and $5 \mathrm{C}$ show clearly that all observable and derived (such as $B_{\text {surf }}$ ) quantities for the Crab, PSR 0540-69, and PSR 1509-58 can be reproduced within our model. Note that the calculated values of $B_{\text {surf }}$ exactly match the corresponding values derived from the standard formula for the magnetic dipole losses in which the stellar radius and moment of inertia are taken to be equal to those the NS model used for each particular pulsar.

\subsection{Effects of Cooling and Stellar Structure}

Figure $5 \mathrm{E}$ shows that with the fast neutrino cooling $\tau_{s d}$ exceeds $t_{\text {model }}$ for a longer time than in the case of slow cooling, but the detailed behavior depends also on other quantities such as the $n$ and $t_{\text {model }}$. The requirement that the model fits the age of the pulsar (besides $P, \tau_{s d}$, and $n$ ) thus puts strong constraints on the thermal evolution of the NS. In the cases of the Crab and PSR 0540-69, such a fit can only be achieved by employing fast neutrino cooling, which results in a rapid freezing of the stellar crust and corresponding decrease (see 
also MP) in the electrical resistivity of the crust. This is clearly seen in Figure 5A: after $t \sim 20 \mathrm{yr}$, the rate of growth of the surface magnetic field slows down substantially, and the magnetic field enters the saturation regime. The Crab pulsar has been claimed several times (see, e.g., Tsuruta 1986; Page \& Baron 1990) to be a typical example of an NS that follows the standard cooling scenario. However, there is actually no real observational evidence in favor of these claims (Page 1994, §6.2) and this pulsar may perfectly well have undergone fast neutrino cooling as we need here. In the case of PSR 0540-69, the progenitor mass is estimated to be around 15-20 $M_{\odot}$ (Kirshner et al. 1989). The latter implies a high mass NS, for which a fast cooling (needed in our analysis to fit the data) is almost inevitable. In the case of PSR 1509-58 there is no such age constraint. However, if our model is correct, then the fit of $P, \tau_{s d}$, and $n$ is evidence in favor of slow neutrino cooling, which, in its turn, would argue for a progenitor less massive than that of the Crab [which is estimated to be about $9 M_{\odot}$ (see, e.g., Nomoto 1985)].

Our conclusion concerning the cooling, fast for the Crab and PSR 0540-69 and slow for PSR 1509-58, is quite robust within the framework of our study. However, our analysis cannot discriminate between different fast cooling scenarios owing, e.g., to the kaon or pion condensates or direct Urca from nucleons and/or hyperons. Note also that our calculations are less sensitive to the stellar radius, and the complexity of internal field (the presence of higher order multipoles, toroidal component, etc.) may also allow us to relax somewhat the constraints on the crust thickness.

\subsection{Predictions for the Crab and SN 1987A}

The detection of thermal emission from the surface of the NS in the Crab can, in principle, establish the occurrence of the fast neutrino cooling for this star in the past. The new ROSAT upper limit (Becker \& Ashenbach 1995), based on the absence of detection, is already marginally inconsistent with the standard cooling scenarios. Our cooling scenario implies an effective temperature well below that in the standard scenario which may potentially be discriminated by the future X-ray observatories. Another interesting point is that, as one can see in Figure $5 \mathrm{~B}$, our calculations imply that the spin-down luminosity of the Crab has been decreasing only slightly during the past centuries. The comparison of the present remnant luminosity with earlier observations may confirm or rule out this possibility. We have used an NS model of mass $1.45 M_{\odot}$ for the Crab which is required by the need for fast cooling within our TPL EOS. However, this precise value of mass

should not be taken too seriously, since the critical mass for the kaon condensation is model dependent, and the value of $a_{3} m_{s}$ we have adopted in our calculations is arbitrary. Owing 
to the estimated low mass of the Crab progenitor (Nomoto 1985) one may suggest a lower mass for the associated NS. In this case, in terms of NS modeling, we would simply need to increase $\left|a_{3} m_{s}\right|$ to reduce the critical mass for kaon condensation, without affecting seriously the maximum mass (which can also be adjusted by changing the underlying baryonic EOS). Thus, our estimate of the NS mass for the Crab is not warranted.

The development of our model was triggered by the announcement of the discovery of an optical pulsar in the remnant of SN 1987A. This detection has been neither confirmed nor ruled out by Hubble Space Telescope observations (Percival et al. 1995). Our original implication ( MP) of the calculations of the field growth for the possible NS in the SN 1987A was based on a stellar model with slow neutrino cooling and resulted in a slow increase of the pulsar's spin-down luminosity, similar to our present model for PSR 1509-58. This first scenario may explain the lack of evidence of powering of the remnant by the pulsar and predicts that this powering should be detectable in a few decades or centuries. However, our modeling of PSR 0540-58, whose progenitor seems to be a twin of the progenitor of SN 1987A (Kirshner et al. 1989), argues for fast neutrino cooling in the case of NS 1987A. If this very young NS follows similar evolution as that in PSR 0540-58, its spin-down luminosity should already be quite high, and it will be growing up by an order of magnitude during the next decade or so and may become detectable. If, however, the magnetic field of the NS 1987A has been submerged down to deeper layers than in the case of PSR 0540-58, then the field growth will be retarded, and the pulsar will be hidden within the remnant for a much longer time. The other possibility is, of course, that the NS 1987A did go into a black hole (Brown, Bruenn, \& Wheeler 1992). This scenario puts strong constraints on the maximum NS mass,$\sim 1.56 M_{\odot}$ (Bethe \& Brown 1995), to which our TPL EOS has been adjusted. Also, the scenario of Brown et al. (1992) stipulates that the mass of the NS 1987A only slightly exceeded this maximum mass. The fact that the NS in PSR 0540-58 (a probable twin of the NS 1987A) did survive both reinforces their suggestion and may imply that it has a mass just below the maximum mass and has undergone a phase of fast neutrino cooling.

\section{SUMMARY AND CONCLUSIONS}

We have presented numerical calculations of early magnetic and spin evolutions of an NS born with a magnetic flux initially trapped under the stellar surface. We have demonstrated explicitly that our calculations may be relevant to the magnetic and spin evolutions of the NSs in PSR 0531+21 (Crab), PSR 0540-69, and PSR 1509-58.

Our principal findings are as follows. 
1. The braking indices of the Crab pulsar $(n=2.5)$, PSR 0540-69 $(n=2.0)$, and PSR 1509-58 ( $n=2.8$ ) can be understood easily in terms of the standard mechanism of magnetic dipole braking, provided that the large scale surface magnetic field of the associated NSs has been growing up with the rate corresponding to the ohmic diffusion of an internal large scale magnetic field, initially submerged under the stellar surface.

2. We account for the existing difference $(\sim 300 \mathrm{yr})$ between the spin-down and real ages for the Crab pulsar. Also, according to our calculations, the real age of PSR 0540-69 is about $890 \mathrm{yr}$, i.e., $770 \mathrm{yr}$ less than its spin-down age, in agreement with the estimated age of the associated SNR 0540-69. Finally, the real age of PSR 1509-58, in contrast to the Crab and PSR 0540-69, may exceed its spin-down age by about 300 yr.

3. In our model the NSs in the Crab, PSR 0540-69, and PSR 1509-58 have initial spin periods of, respectively, $P_{0}=21,39$, and $20 \mathrm{~ms}$, and their initial surface magnetic fields are in the range of $(1-5) \times 10^{10} \mathrm{G}$.

4. The spin-down luminosity of the Crab, PSR 0540-69, and PSR 1509-58 reaches its maximum at an NS age of $\sim 140, \sim 250$, and $\sim 80 \mathrm{yr}$, respectively.

5. Our analysis may sensibly indicate that NSs in the Crab and PSR 0540-69 have thin $(\lesssim 200 \mathrm{~m})$ outer crusts and have experienced a phase of fast neutrino cooling. In contrast, the NS in PSR 1509-58 probably has a relatively thick $(\sim 430 \mathrm{~m})$ outer crust, and has undergone a slow neutrino cooling.

To extend our investigation of the early magnetic and spin evolution of an NS to other young pulsars, we need to have reliable measurements of their braking indices that are not available at the moment. However, the observations recently initiated with the ROSAT X-ray telescope (see, e.g., Aschenbach, Egger, \& Trümper 1995) of fine structures in young supernova remnants (SNRs) containing pulsars may provide us with more accurate estimates of the real ages of young radio pulsars associated with the SNRs. Given such estimates, we would be able to proceed farther with a more extensive analysis of the effect under consideration in pulsars.

Finally, a more detailed study should incorporate some other possible mechanisms mentioned in the introduction. Spin-up of an NS by early neutrino emission can be incorporated in our model since it is a well defined mechanism and requires the same ingredients of stellar structure and cooling. The various mechanisms reducing $n$ below 3 are very model dependent and would increase the number of free parameters. Quadrupolar radiation, magnetic and/or gravitational (resulting in $n>3$ ) can reasonably be excluded. The crustal "plate" motion is of major uncertainty and is practically impossible to model quantitatively. Although plate tectonics is hypothetical, crust cracking ("plate tectonic" 
activity ?) seems to be taking place in the Crab (Link, Epstein, \& Baym 1992; Alpar et al. 1994), and crustal plates may manifest themselves at the surface of the Geminga NS (Page, Shibanov, \& Zavlin 1995).

A.M. thanks the Alexander-von-Humboldt-Stiftung for support. At UNAM, this work has been supported by a UNAM-DGAPA grant IN105794 and a Cátedra Patrimonial from Conacyt. The authors also thank an anonymous referee for useful comments, which have improved the clarity of the manuscript. 
Table 1. Characteristics of Pulsars and Parameters of Models ${ }^{\text {a }}$

\begin{tabular}{cccccccccc}
\hline \hline PSR & $P$ & $n$ & $\begin{array}{c}\tau_{s d} \\
(\mathrm{yrs})\end{array}$ & $\begin{array}{c}\tau_{S N R} \\
(\mathrm{yrs})\end{array}$ & $\begin{array}{c}t_{\text {model }} \\
(\mathrm{yrs})\end{array}$ & $\begin{array}{c}P_{0} \\
(\mathrm{~ms})\end{array}$ & $\begin{array}{c}\text { Mass } \\
M_{\odot}\end{array}$ & $\begin{array}{c}z_{\text {sub }} \\
(\mathrm{m})\end{array}$ & $\begin{array}{c}\rho_{\text {sub }} \\
\left(10^{10} \mathrm{~g} / \mathrm{cm}^{3}\right)\end{array}$ \\
\hline $0540-69$ & 50.4 & $2.01(2)^{\mathrm{b}}$ & 1660 & $760 \pm 100^{\mathrm{c}}$ & 890 & 39 & 1.50 & $85-90$ & $2.62-3.16$ \\
$0531+21$ & 33.4 & $2.509(1)^{\mathrm{d}}$ & 1260 & $810 \pm 100^{\mathrm{c}}$ & 940 & 21 & 1.45 & $95-100$ & $1.94-2.34$ \\
$1509-58$ & 150.2 & $2.837(1)^{\mathrm{e}}$ & 1549 & $1810^{\mathrm{f}}$ & 1860 & 20 & 1.35 & $170-175$ & $1.43-1.56$ \\
\hline
\end{tabular}

${ }^{a}$ Listed are: pulsar spin period $P$, braking index $n$, and spin-down age $\tau_{s d}$; associated supernova remnant age $\tau_{S N R}$; model age $t_{\text {model }}$ of the NS (as used in our calculations) and initial pulsar spin period $P_{0}$; theoretical mass of the NS; depth $z_{s u b}$ and density $\rho_{s u b}$ of initial submergence of the magnetic field below the surface.

${ }^{\text {b} N a g a s e ~ e t ~ a l . ~ 1990 ; ~ G o u i f f e s ~ e t ~ a l . ~} 1992$.

${ }^{\mathrm{c}}$ Kirshner et al. 1989.

d'Lyne et al. 1988.

'Kaspi et al. 1994.

${ }^{\mathrm{f}} \mathrm{SN}$ 185: Thorsett 1992. 


\section{REFERENCES}

Ainsworth, T. L., Wambach, J. \& Pines, D. 1989, Phys. Lett., B222, 173

Alpar, M. A., Chau, H. F., Cheng, K. S., \& Pines, D. 1994, ApJ, 427, L29

Alpar, M. A., \& Ögelman, H. 1990, ApJ, 349, L55

Aschenbach, B., Egger, R., \& Trümper, J. 1995, Nature, 373, 587

Becker, W., \& Aschenbach, A. 1995, in The Lives of the Neutron Stars, ed. A. Alpar, Ü Kiziloğlu, \& J. van Paradijs (Dordrecht: Kluwer), 47

Bethe, H. A. \& Brown, G. E. 1995, ApJ, 445, L129

Blandford, R. D., Applegate, J. H., \& Hernquist, L. 1983, MNRAS, 204, 1025

Blandford, R. D., \& Romani, R. W. 1988, MNRAS, 234, 57p

Brown, G. E., \& Bethe, H. A. 1994, ApJ, 423, 659

Brown, G. E., Bruenn, S. W., \& Wheeler, J. C. 1992, Comments Astrophys., 16, 153

Brown, G. E., Kubodera, K., Page, D., \& Pizzochero, P. 1988, Phys. Rev., D37, 2042

Brown, G. E., \& Rho, M. 1995, Phys. Rep., in press (e-print hep-ph/9504250)

Chanmugam, G., \& Sang, Y. 1989, MNRAS, 241, 295

Friedman, B., \& Pandharipande, V. R. 1981, Nucl. Phys., A361, 502

Gouiffes, Ch., Finley, J. P., \& Ögelman, H. 1992, ApJ, 394, 581

Kaspi, V. M., Manchester, R. N., Siegman, B., Johnston, S., \& Lyne, A. G. 1994, ApJ, 422, L83

Kirshner, R.P., Morse, J.A., Winkler, P.F., \& Blair, W.P. 1989, ApJ, 342, 260

Lattimer, J. M., Pethick, C. J., Prakash, M., \& Haensel, P. 1991, Phys. Rev. Lett., 66, 2701

Levenfish, K. P., \& Yakovlev, D. G. 1994a, Astron. Rep., 71, 247

— 1994b, Astron. Lett., 20, 43

Link, B., Epstein, R. I., \& Baym, G. 1992, ApJ, 390, L21

Lyne, A. G., Pritchard, R. S., \& Smith, F. G. 1988, MNRAS, 233, 667

Manchester, R. N., \& Taylor, J. H. 1977, Pulsars (San Fransisco: Freeman) (MT)

Michel, F. C. 1991, Theory of Neutron Star Magnetospheres (Chicago: Univ. Chicago Press)

- 1994, MNRAS, 267, L4: (M94)

Muslimov, A., \& Page, D. 1995, ApJ, 440, L77 (MP) 
Müther, H., Prakash, M., \& Ainsworth, T. L. 1987, Phys. Lett., B199, 469

Nagase, et al. 1990, ApJ, 351, L13

Nomoto, K. 1985, in The Crab Nebula and Related Supernova Remnants, ed. M. C. Kafatos \& R. B. C. Henry (Cambridge: Cambridge University Press), 97

Page, D. 1994, ApJ, 428, 250

Page, D., \& Applegate, J. H. 1992, ApJ, 394, L17

Page, D., \& Baron, E. 1990, ApJ, 354, L17

Page, D., Shibanov, Yu. A., \& Zavlin, V. E. 1995, Ap. J. , 451, L21

Percival, J. W., et al. 1995, ApJ, 446, 832

Pethick, C. J. 1992, Rev. Mod. Phys., 64, 1133

Prakash, M., Ainsworth, T. L. \& Lattimer, J. M. 1988, Phys. Rev. Lett., 61, 2518 (PAL)

Ruderman, M. 1991, ApJ, 382, 576

Strom, R. G. 1994, MNRAS, 268, L5

Takatsuka, T. 1972, Prog. Theor. Phys., 48, 1517

— 1973, Prog. Theor. Phys., 50, 1754

Tatsumi, T. 1995, Prog. Theor. Phys. Suppl., 120, in press

Thorsett, S. E. 1992, Nature, 356, 690

Thorsson, V., Prakash, M., \& Lattimer, J. 1994, Nucl. Phys. A572, 693 (TPL)

Thorsson, V., Prakash, M., Tatsumi, T., \& Pethick, C. J. 1995, Phys. Rev. D, submitted (e-print nucl-th/9502004)

Tsuruta, S. 1986, Comments Astrophys., 11, 151

Urpin, V. A., Levshakov, S. A., \& Yakovlev, D. G. 1986, MNRAS, 219, 703

van Kerkwijk, M. H., van Paradijs, J. \& Zuiderwijk, E. J. 1995, A\&A, in press (e-print astro-ph/9505071)

This preprint was prepared with the AAS IATEX macros v4.0. 
Fig. 1.- Mass-radius relationships for the four NS EOSs: FP (Friedman \& Pandharipande 1981), MPA (Müther et al. 1987), PAL (Prakash et al. 1988: see text for the specific parameters used), and TPL (Thorsson et al. 1994: see text for the specific parameters used). The regions in which $M$ decreases with $R$ are unstable, so that for the TPL EOS the branch b-c collapses onto the branch $d-c$, and the branch $a-b$ is probably not realized in nature and also collapses onto c-d. As a result, for the TPL EOS the normal NSs can be produced for gravitational masses below $1.37 M_{\odot}$ (point a), while above this mass the leap into the regime of formation of nuclear stars (branch c-e with kaon condensate) occurs. Point e denotes the maximum mass of $1.575 M_{\odot}$ corresponding to this EOS.

Fig. 2.- Density profiles for five neutron-nuclear stars from our TPL EOS. At the kaon condensation phase transition the density jumps from $\rho_{1}=1.03 \times 10^{15} \mathrm{~g} \mathrm{~cm}^{-3}$ to $\rho_{2}=1.76 \times 10^{15} \mathrm{~g} \mathrm{~cm}^{-3}$ and, owing to the enormous amount of kaons present, the proton fraction jumps from $9.85 \%$ at $\rho_{1}$ up to $54 \%$ at $\rho_{2}$, while the electron fraction drops from $6.14 \%$ down to $0.0022 \%$. Despite the large proton fraction and owing to the very low electron fraction the direct Urca process is forbidden at densities above $\rho_{2}$ and is allowed only when $\rho$ reaches $\rho_{3}=2.8 \times 10^{15} \mathrm{~g} \mathrm{~cm}^{-3}$. See TPL for more details.

Fig. 3.- Cooling curves for five stellar models with the TPL EOS. The $1.35 M_{\odot}$ star cools according to the "standard" scenario, the 1.40 and $1.45 M_{\odot}$ stars undergo fast neutrino cooling from the kaon condensate, and the two heaviest stars allow also the direct Urca process (see Fig. 2) and cool even faster. The ordinate is the redshifted effective temperature.

Fig. 4.- Profiles of the internal temperature for two cooling stars with the TPL EOS. The profiles for the $1.40,1.50$, and $1.55 M_{\odot}$ stars are similar to the $1.45 M_{\odot}$ case.

Fig. 5.- Plot of (A) the surface value of the polar magnetic field strength, (B) spin-down luminosity, (C) spin period, (D) braking index, and (E) spin-down age vs. the model age of the NS. Dots on the curves correspond to the "empirical" and derived data for these pulsars (see $\S 3$ for details). The parameter value $L_{0}$ in $(\mathrm{B})$ is equal to $2.4 \times 10^{34}, 1.4 \times 10^{33}$, and $4.2 \times 10^{35} \mathrm{erg} \mathrm{s}^{-1}$ for the Crab, PSR 0540-69, and PSR 1509-58, respectively. The dependence $\tau_{s d}=t_{\text {model }}$ is shown by the dotted line in $(\mathrm{E})$. 


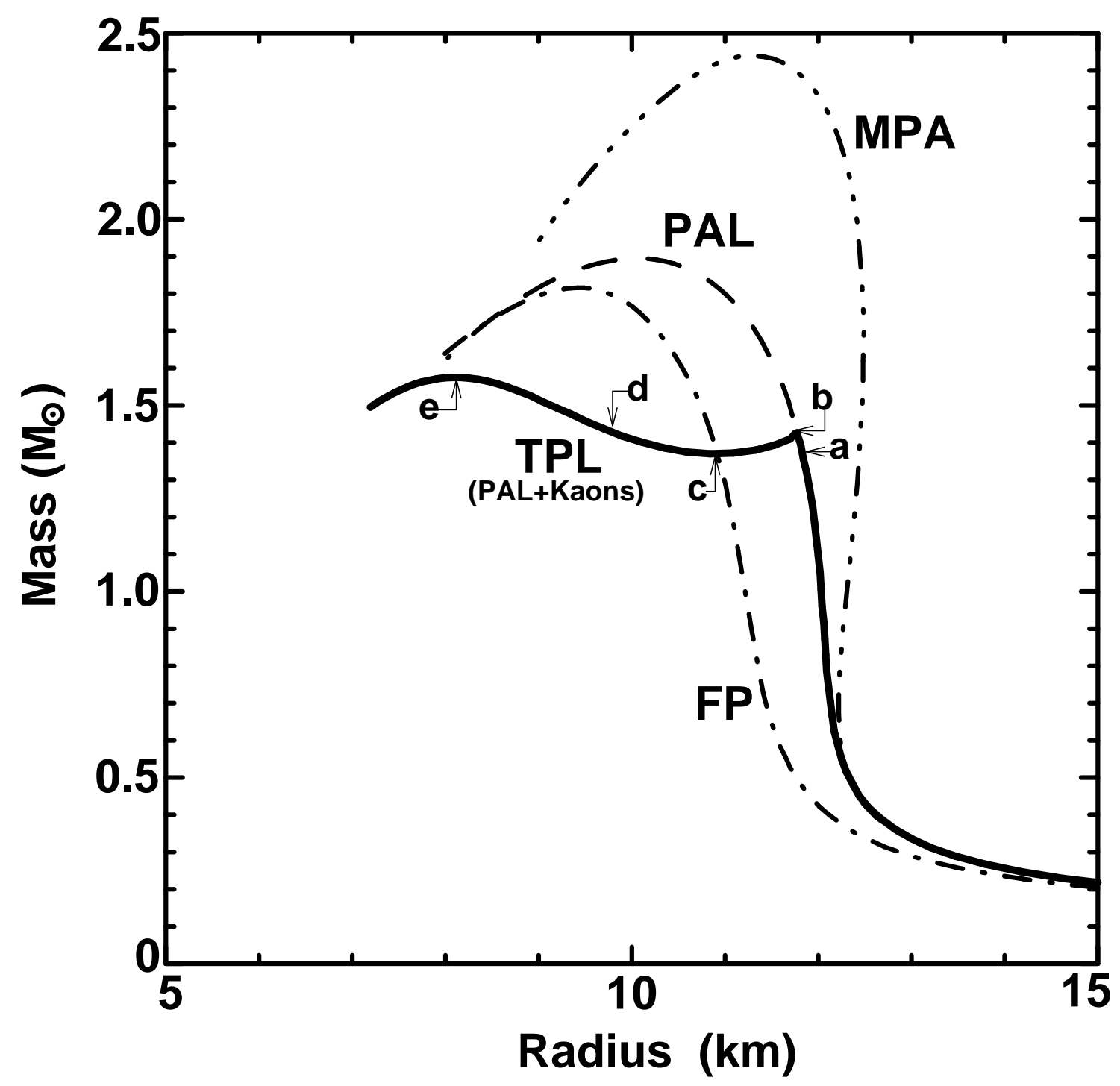

Muslimov \& Page

Figure 1 


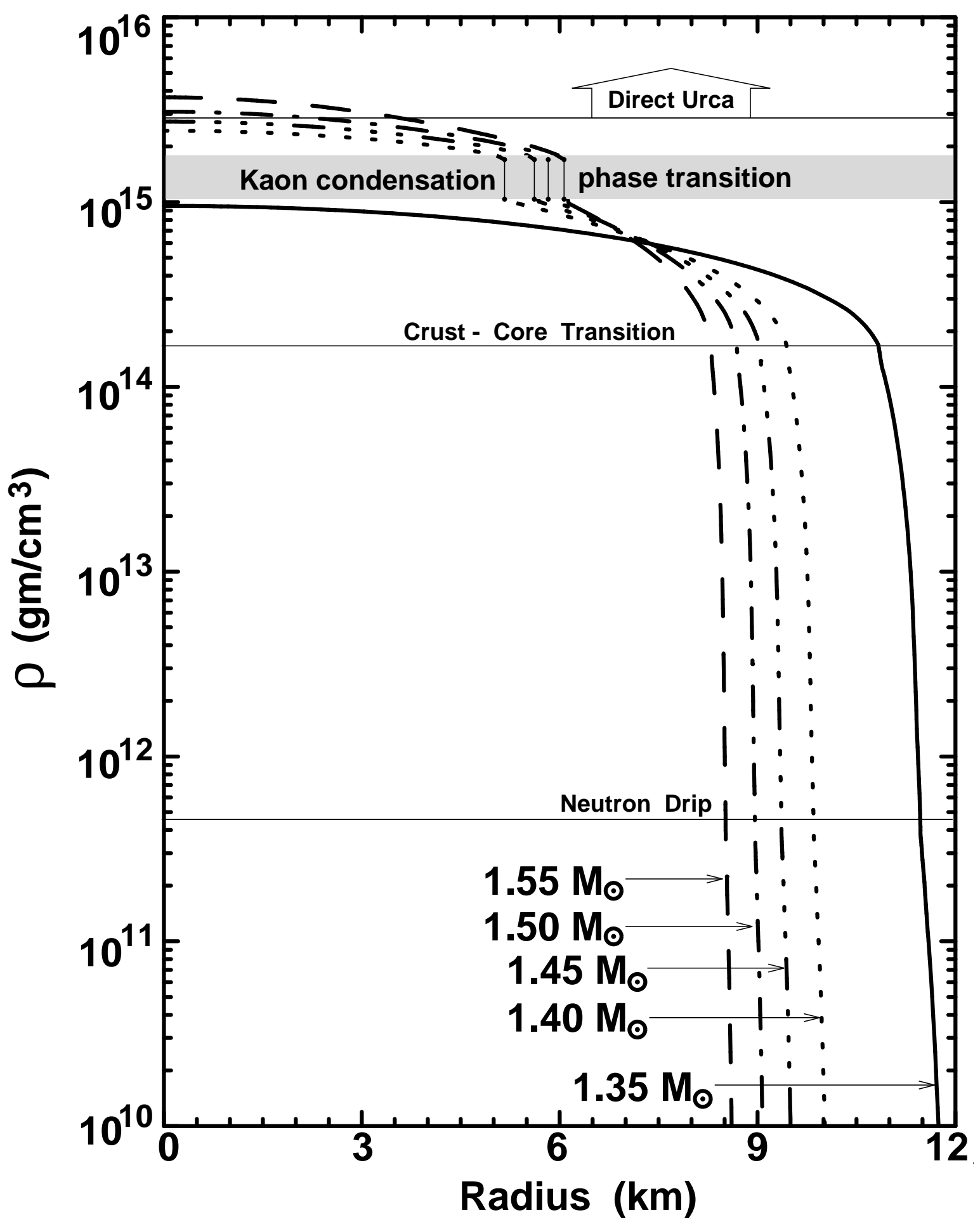

Muslimov \& Page

Figure 2 


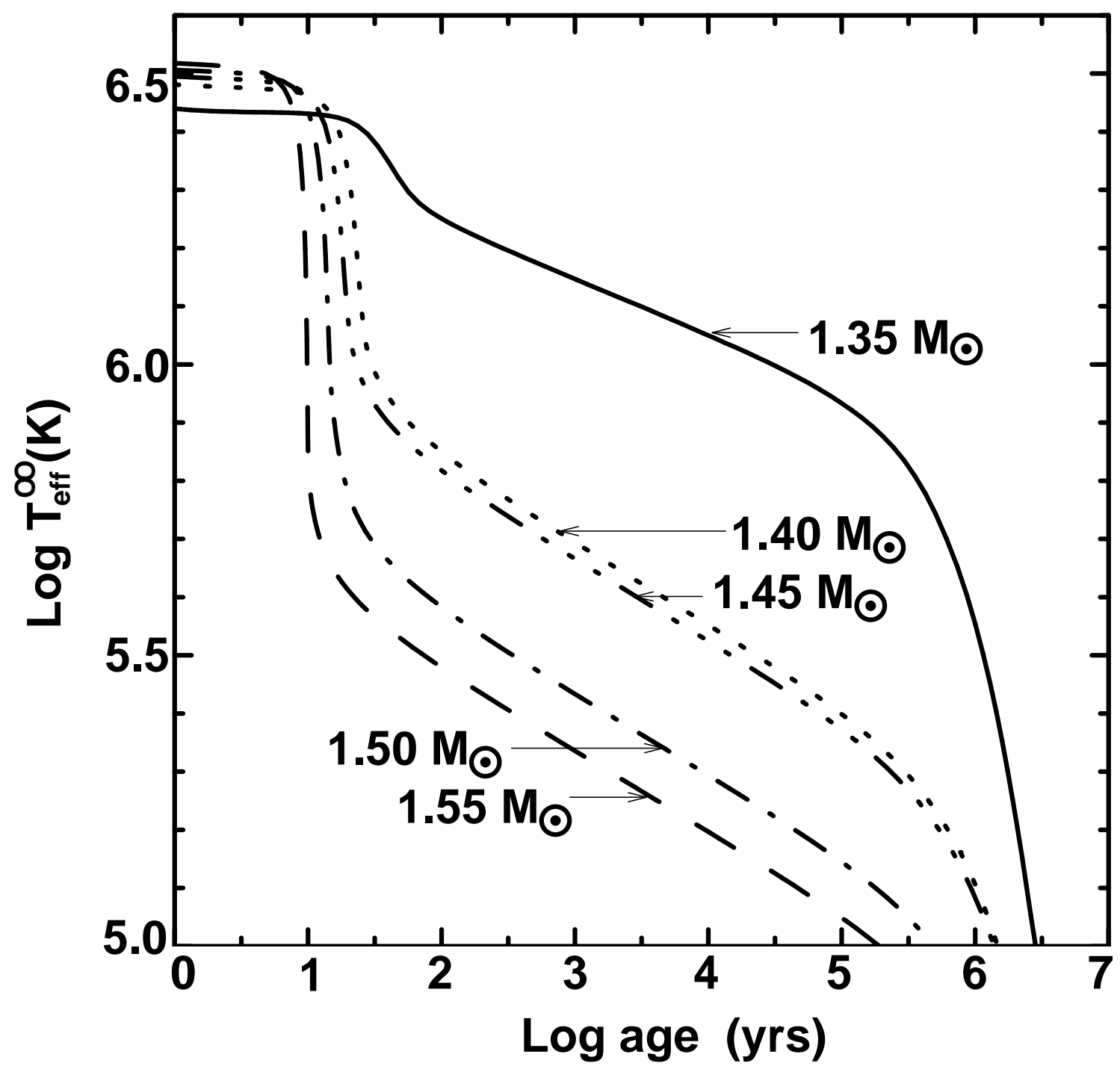

Muslimov \& Page

Figure 3 


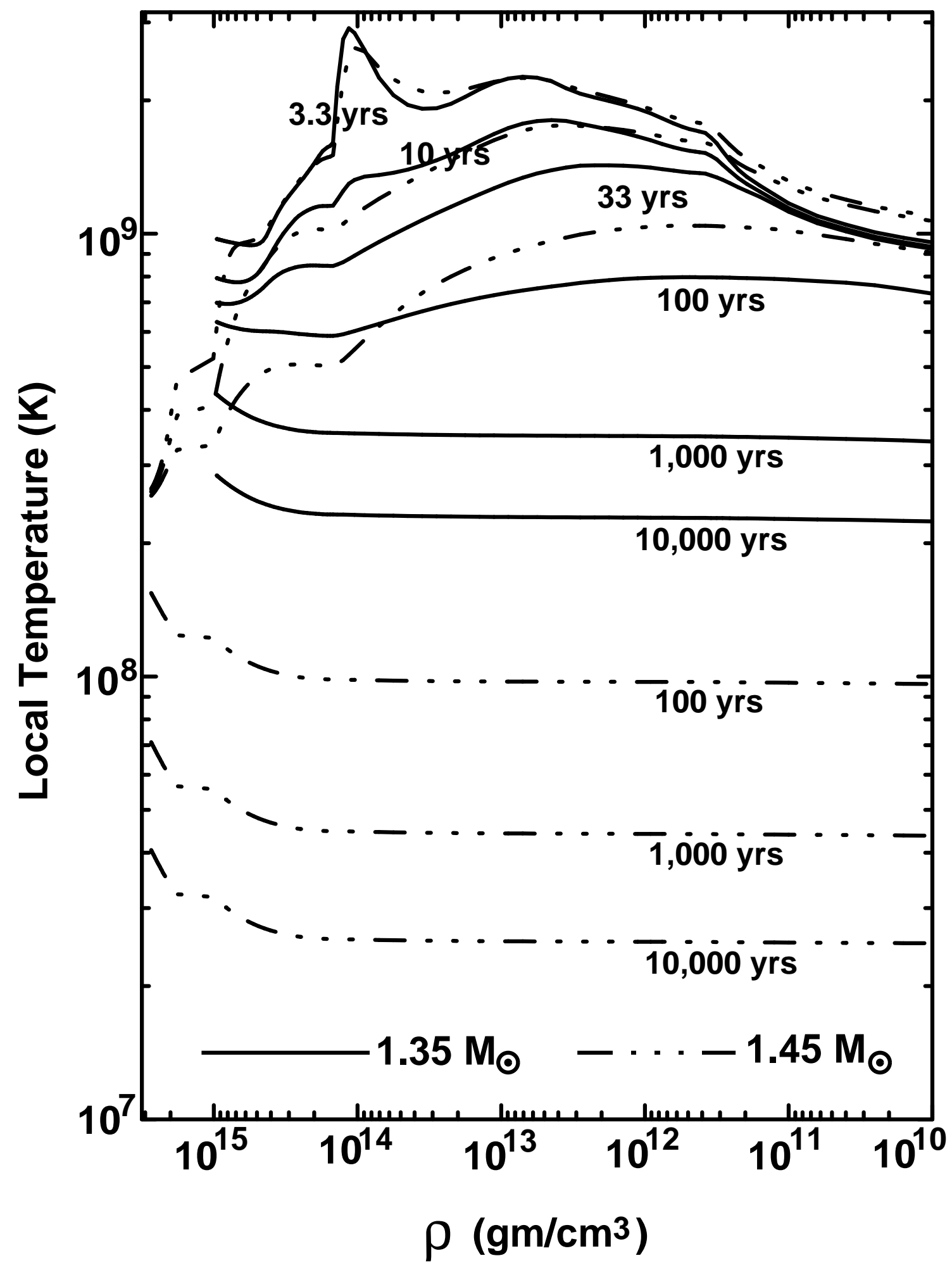

Muslimov \& Page

Figure 4 


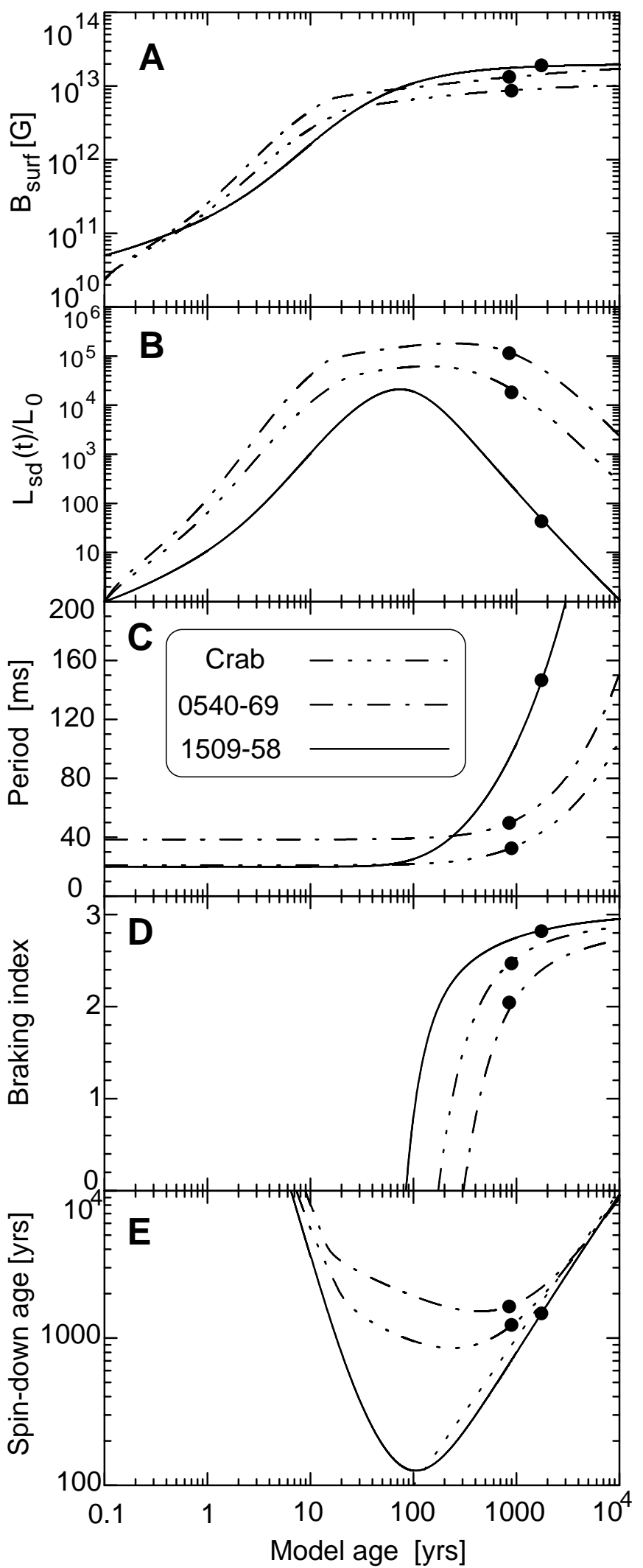

Muslimov \& Page $\quad$ Figure 5 